\title{
How I Manage Thrombotic/Thromboembolic Complications in Myeloproliferative Neoplasms
}

\section{How I treat: Thrombotische/thromboembolische Komplikationen bei Patienten mit Myeloproliferativen Neoplasien}

\author{
Steffen Koschmieder ${ }^{1}$ \\ ${ }^{1}$ Department of Medicine (Hematology, Oncology, Hemostaseology, \\ and Stem Cell Transplantation), Faculty of Medicine, RWTH Aachen \\ University, Aachen, Germany \\ Hämostaseologie 2020;40:47-53.

\begin{abstract}
Address for correspondence Prof. Dr. med. Steffen Koschmieder, Klinik für Hämatologie, Onkologie, Hämostaseologie und Stammzelltransplantation, Medizinische Fakultät, Uniklinik RWTH Aachen, Pauwelsstr. 30, D-52074 Aachen, Germany
\end{abstract} \\ (e-mail: skoschmieder@ukaachen.de).
}

\author{
Abstract \\ Keywords \\ - myeloproliferative \\ neoplasms (MPNs) \\ - thrombosis/ \\ thromboembolism \\ - therapy \\ - anticoagulation \\ - cytoreduction \\ - polycythemia vera (PV) \\ - essential \\ thrombocythemia (ET) \\ - primary myelofibrosis \\ (PMF)
}

\section{Zusammenfassung}

\section{Schlüsselwörter \\ - Myeloproliferative Neoplasien (MPN) \\ - Thrombosen/ Thromboembolien \\ - Therapie \\ - Antikoagulation \\ - Zytoreduktion}

Patients with myeloproliferative neoplasms (MPNs), such as polycythemia vera, essential thrombocythemia, and primary myelofibrosis, are at increased risk for arterial and venous thrombosis/thromboembolism. In particular, the risk of splanchnic venous thrombosis, such as portal vein thrombosis or Budd-Chiari syndrome, is significantly higher in patients with MPN than in the normal population. At the same time, MPN patients are at increased risk for severe bleeding. Therefore, the treatment of patients with MPN must be based on their suspected probability of thrombosis/thromboembolism and bleeding. For this purpose, patient and MPNspecific risk factors are used. Patients at expected high risk of thrombosis should receive adequate primary or secondary thromboprophylaxis in addition to cytoreductive therapy. This may consist of antiplatelet agents and/or anticoagulant agents and must be balanced with the individual bleeding risk. The goal is to increase the quality of life and life span of patients with MPNs by preventing (re-)thrombosis and severe bleeding.

Patienten mit Myeloproliferativen Neoplasien (MPN), wie der Polycythämia vera (PV), Essentiellen Thrombozythämie (ET) und Primären Myelofibrose (PMF), sind einem erhöhten Risiko für arterielle und venöse Thromboembolien ausgesetzt. Besonders das Risiko für splanchnische Venenthrombosen, wie die Pfortaderthrombose oder das Budd-Chiari-Syndrom, ist bei Patienten mit MPN vielfach höher als in der Normalbevölkerung. Gleichzeitig besteht bei MPN-Patienten ein erhöhtes Risiko für schwergradige Blutungen. Daher muß sich die Therapie der Patienten mit MPN an der Wahrscheinlichkeit für Thromboembolien und Blutungen orientieren. Hierfür werden patienten- und MPN-spezifische Risikofaktoren herangezogen. Patienten mit zu erwartendem hohem Risiko für Thrombosen sollten zusätzlich zu einer zytoreduktiven Therapie eine angemessene Primär- oder Sekundärprophylaxe erhalten. Diese kann received

July 23, 2019

accepted after revision

December 15, 2019 (c) 2020 Georg Thieme Verlag KG Stuttgart · New York
DOI https://doi.org/

10.1055/s-0040-1701474. ISSN 0720-9355. 
- Polycythämia vera (PV)

- Essentielle

- Thrombozythämie (ET)

- Primäre Myelofibrose (PMF) aus Thrombozytenfunktionshemmern und/oder Antikoagulanzien bestehen und muß mit dem individuellen Blutungsrisiko balanciert werden. Ziel ist es, durch die Verhinderung von (Re-)Thrombosen und schwergradigen Blutungen die Lebensqualität und Lebensspanne der Patienten mit MPN zu erhöhen.

\section{Introduction}

Philadelphia-negative myeloproliferative neoplasms (MPNs) are chronic hematological malignancies. They share a common pathogenesis, which derives from the JAK-STAT pathway activation through the presence of a JAK2, calreticulin (CALR), or the myeloproliferative leukemia virus oncogene (MPL) mutation in the hematopoietic stem and progenitor cells (HSPCs) and extramedullary hematopoiesis causing splenomegaly. The classical Ph neg MPN subtypes include polycythemia vera (PV), essential thrombocythemia (ET), and primary myelofibrosis (PMF), and their diagnosis criteria have been revised by the World Health Organization (WHO) in 2016. ${ }^{1}$ Common symptoms include those generally found in MPN such as fatigue, early satiety and abdominal discomfort, inactivity, concentration problems, constitutional symptoms (fever, night sweats, and weight loss), bone pain, but also aquagenic pruritus, which designates pruritus upon contact with water. ${ }^{2}$ And during the course of disease, patients may acquire complications such as thrombotic/ thromboembolic events, severe hemorrhage, or disease progression to post-PV-myelofibrosis (post-PV-MF), or post-ETmyelofibrosis (post-ET-MF), or even acute leukemia. Treatment of patients with MPN aims at preventing these complications through various cytoreductive agents and disease modulation. However, these therapies are noncurative, and while allogeneic stem cell transplantation (SCT) may be curative, this option is associated with significant morbidity and mortality and is thus reserved for the minority of patients with a poor prognosis.

Patients who have encountered thrombosis/thromboembolism or severe bleeding before or during the course of an MPN are at high risk for recurrent thrombosis/thromboembolism. Real-world analyses from MPN registries have found the frequency of thrombosis/thromboembolism and major bleeding events in MPN patients to be 11 to $33 \%$ and 2 to $8 \%$, respectively. The location of the thrombotic events differed significantly among the MPN subtypes PV, ET, and PMF. ${ }^{3}$ In particular, splanchnic vein thrombosis, including portal vein thrombosis and Budd-Chiari syndrome, is by far more frequent in MPN than in non-MPN patients. ${ }^{4}$ Mechanisms of thrombosis in MPN include increased numbers and activation of blood cells and endothelial cells, ${ }^{5}$ JAK2 V617Finduced inflammation ${ }^{6}$ and integrin-mediated adhesiveness, ${ }^{7}$ as well as tissue factor-containing microparticles, ${ }^{8}$ and activation of procoagulant factors ${ }^{9}$ (all reviewed in Kroll et $\mathrm{al}^{10}$ ). In addition, neutrophil extracellular trap formation has been implicated in thrombosis/thromboembolism development in mice and MPN patients expressing JAK2V617F. ${ }^{11}$

Therefore, since MPNs are chronic diseases, the requirement for anticoagulant and/or antiplatelet treatment is typically lifelong. And in patients with thrombosis/thromboembolism in whom no proper cause can be found, an MPN needs to be ruled out, especially if they also have abnormal blood cell counts and/ or splenomegaly. The incidence of thrombosis/thromboembolism peaks around the diagnosis of an MPN, suggesting that the thrombosis/thromboembolism itself often leads to the MPN diagnosis. $^{3}$ Conversely, the incidence of severe bleeding is low before the MPN diagnosis but is increased thereafter, ${ }^{3}$ which suggests that therapy of MPN and/or progression contributes to bleeding in these patients. In the following, I summarize my approach to the management of thrombosis/thromboembolism in patients with MPN, including specific recommendations for PV and ET ( - Fig. 1). Current treatment recommendations have recently been revised (see www.onkopedia.com/de and ELN recommendations ${ }^{12}$ ). In addition to these specific recommendations, cessation of smoking and adequate physical exercise should be advised to all MPN patients.

\section{Polycythemia Vera}

PV is characterized by expansion of all three hematopoietic lineages in the bone marrow (granulocytic, erythrocytic, and megakaryocytic) and peripheral blood (neutrophilia, erythrocytosis, and thrombocytosis), the presence of a JAK2 mutation, and a subnormal erythropoietin level. Its incidence ranges around $1 / 100,000$ inhabitants, ${ }^{13}$ but its prevalence is considerably greater due to the long course of the disease in most patients. The exon $14 J A K 2$ mutation is present in approximately $96 \%$ of patients and translates to the mutant JAK2V617F oncoprotein. The exon 12 JAK2 mutation is present in roughly 2 to $3 \%$ of patients and is associated with a more exclusive expansion of red cell mass, also termed polycythemia vera rubra. Common symptoms include those generally found in MPNs (see above), with aquagenic pruritus being more frequent in PV than in the other MPN subtypes. ${ }^{2}$ The risk of progression to post-PV-MF and acute leukemia is 9 to $21 \%$ and 3 to $10 \%$, respectively, ${ }^{14}$ and mean survival of PV patients ranges from 10.8 to 27.8 years. ${ }^{15}$

Thrombosis/thromboembolism is a major cause for morbidity and mortality in PV patients. ${ }^{14}$ The major risk factors for thrombosis/thromboembolism are older age, previous history of thrombosis, leukocytosis, an increased JAK2 V617F allele burden, and certain gene expression signatures. ${ }^{14}$ 


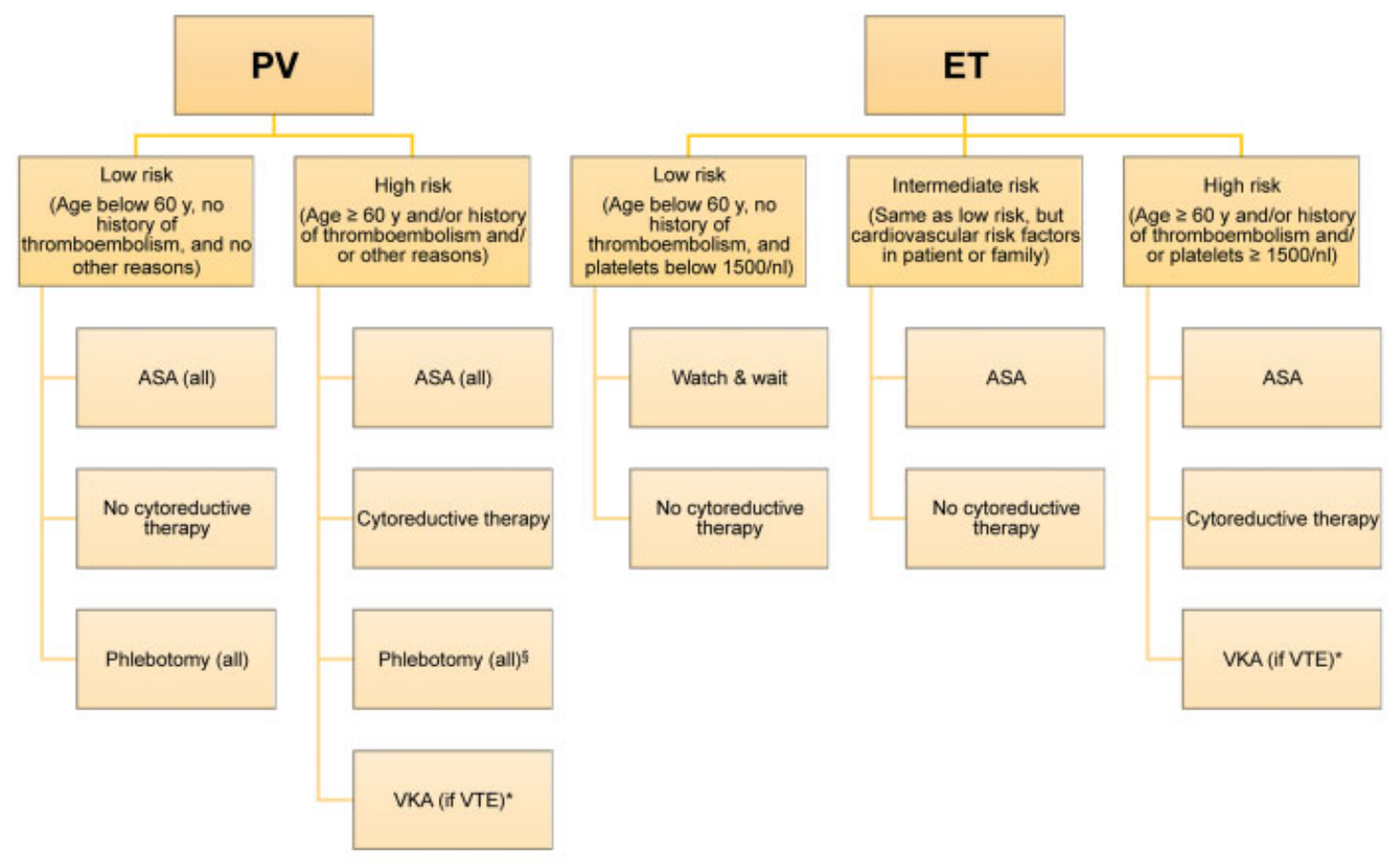

Fig. 1 Recommendations for antiplatelet and anticoagulant therapy as well as cytoreductive treatment in PV and ET. ASA, acetylsalicylic acid; ET, essential thrombocythemia; PV, polycythemia vera; VKA, vitamin K antagonist; VTE, venous thromboembolism. See the text for more detailed explanations. ${ }^{a}$ VKA is the first choice, non-vitamin K antagonists (NOACs) are the second choice; stop ASA if starting VKA or NOACs. ${ }^{b}$ Phlebotomy aims at keeping hematocrit below 45\%; phlebotomies may no longer be necessary after starting cytoreductive therapy.

Controlled randomized clinical trials have demonstrated enhanced thrombosis-free survival for both low-dose acetylsalicylic acid (ASA) $)^{\mathrm{a}}$ treatment $^{16}$ and phlebotomies. ${ }^{17}$ Thus, when the diagnosis of PV is made, all patients should receive both of these therapeutic interventions, unless there are contraindications (which are rare). In addition, the so-called "high-risk" PV patients, i.e., those aged over 60 years and/or those that have experienced a thrombosis/ thromboembolism, should additionally receive cytoreductive treatment. In addition, progression of myeloproliferation (e.g., with development of relevant leukocytosis, thrombocytosis, and/or splenomegaly) or intolerance to phlebotomies may also be indications for cytoreduction. However, both the type of cytoreductive therapy and the anticoagulant agent used for thrombosis/thromboembolism treatment have to be chosen individually according to the patient's needs:

- High-risk PV patients that have not yet experienced any thrombosis/thromboembolism should receive hydroxyurea $^{18}$ or ropeginterferon $\alpha$ (ropegIFNa) $)^{19}$ as first-line cytoreductive treatment (both options currently approved in Europe) or ruxolitinib as second-line treatment. ${ }^{20}$ Further, nonapproved drugs include busulfan and other IFNa compounds. Quite often, phlebotomies will not be necessary anymore when cytoreduction has been initiated. However,

\footnotetext{
according to international convention, the term "low-dose ASA" designates doses between 75 to $100 \mathrm{mg} / \mathrm{d}$.
}

ASA should not be discontinued, even when phlebotomies are no longer necessary.

- High-risk PV patients that have experienced an arterial thrombosis/thromboembolism such as a stroke or myocardial infarction should receive life-long ASA treatment in addition to cytoreductive treatment (see above), along with the usual further secondary prophylaxis (ACE or AT receptor inhibitors, statins, etc.). Vitamin K antagonists (VKAs) may be indicated in such patients, either if they suffer from repeated arterial events despite ASA medication or if they have additional thrombophilic conditions such as antiphospholipid syndrome (APS). Non-vitamin K oral anticoagulants (NOACs) may also be given in patients where VKA cannot be given, but not in those with APS and MPN. NOAC use is currently not recommended in patients with APS, with the European Medicines agency (EMA) issuing a warning (https://www.ema.europa.eu/en/documents/prac -recommendation/prac-recommendations-signals-adop ted-8-11-april-2019-prac-meeting_en.pdf) that "direct acting oral anticoagulants (DOACs) including rivaroxaban/ apixaban/edoxaban/dabigatran etexilate are not recommended for patients with a history of thrombosis who are diagnosed with antiphospholipid syndrome," particularly those with threefold positivity for lupus anticoagulant, anticardiolipin antibodies, and anti- $\beta 2$-glycoprotein antibodies. The most recent summaries of product characteristics of the approved NOACs now also advise against the use of NOACs in all patients with APS.

- High-risk PV patients having experienced venous thrombosis/thromboembolism (VTE) should receive proper 
anticoagulant therapy similar to non-PV patients, in addition to cytoreductive therapy (see above). No evidencebased data exist to inform us about the need to co-administer antiplatelet therapy such as ASA in patients receiving anticoagulant therapy. ${ }^{21}$ However, the European Society for Medical Oncology (ESMO) guidelines specifically recommend to use "oral anticoagulation instead of aspirin," "depending on the thrombosis type" (i.e., in VTE; Table 4 in Vannucchi et $\mathrm{al}^{22}$ ). Importantly, co-administration of antiplatelet agents with VKA increased major bleeding events compared with antiplatelet agents or VKA alone. ${ }^{23}$ Therefore, I typically discontinue ASA during the time of anticoagulant treatment for VTE in patients with PV or ET. The duration of anticoagulant treatment is still a matter of debate. However, recently, recommendations on this issue have been published. ${ }^{24}$ According to these recommendations, extended to life-long anticoagulant treatment should be considered for those PV patients with "high-risk VTE": recurrent VTE, splanchnic vein thrombosis, life-threatening VTE, progressing MPN, and those with a low bleeding risk. ${ }^{24}$ However, in PV patients with provoked VTE and those with unprovoked distal deep vein thrombosis, cessation of anticoagulant therapy can be discussed. ${ }^{25}$ ASA should be reinitiated after cessation of anticoagulant therapy in these cases.

Whether NOACs are equivalent to VKAs in MPN is still unknown and a matter of debate. And while NOACs are certainly used in MPN patients, ${ }^{3}$ and their use will most likely increase in the future, analyses form clinical studies and MPN registries are needed to validate or refute their use in PV and the other MPNs, and recommendations by the Haemostasis Working Party of the German Society of Hematology and Oncology (DGHO), the Austrian Society of Hematology and Oncology (ÖGHO), and Society of Thrombosis and Haemostasis Research (GTH e.V.) have so far favored VKA over NOACs. $^{24}$

\section{Essential Thrombocythemia}

The hallmark of ET is thrombocytosis, coupled with megakaryocytic hyperplasia of the bone marrow and the presence of a clonal marker. Genetic analysis of driver mutations revealed the presence of a JAK2 V617F, CALR, and MPL mutation in up to $50-60,20-25$, and $5 \%$ of ET patients, leaving approximately 10 to $15 \%$ "triple-negative" patients, who have other mutations or chromosomal aberrations. ${ }^{26}$ The incidence of ET has been calculated to be approximately 2-3/ 100,000 inhabitants, a slightly higher rate than previously reported, due to the availability of the genetic markers, allowing exclusion of reactive thrombocytosis cases. ${ }^{13}$ Symptoms include those mentioned in the Introduction, but splenomegaly, itching, and weight loss are less common in ET than in PV and PMF. ${ }^{2}$ Progression to post-ET-MF and acute leukemia is rare, especially when considering "true ET" (ET diagnosed according to the WHO 2016 criteria) cases by separating pre-PMF cases. ${ }^{27}$ Patients with "true ET" have a normal life expectancy. ${ }^{28}$
After making the diagnosis of ET, physicians should estimate the risk of the individual patient with ET (low- vs. intermediate- vs. high-risk patients, as defined by the DGHO for ET; see below), since this will define the treatment recommendation in this patient. Given the normal life expectancy of the patients with ET, this risk in the clinical setting designated the risk for thrombotic and severe bleeding events.

Low-risk ET patients, as defined by the DGHO (i.e., those aged $\leq 60$ years, without previous thrombosis/thromboembolism or severe hemorrhage, and in whom platelet counts have remained below $1,500 / \mathrm{nL}$ ), typically do not need ET-specific treatment, and an approach of watchful waiting is indicated. The use of antiplatelet agents has been the matter of some debate. ${ }^{29,30}$ However, neither the current German recommendations on the management of ET by the DGHO (www.onkopedia.com/de), nor the European LeukemiaNet (ELN) recommendations, ${ }^{12}$ nor the NCCN guidelines ${ }^{21}$ favor the general use of ASA in all patients with ET. Therefore, accordingly, I typically do not use ASA in most low-risk patients with ET. Conversely, "all patients with ET should be managed with lowdose aspirin if microvascular disturbances are present,"12 and this is also my current practice.

Intermediate-risk ET patients, as defined by the DGHO, are those who have first-degree relatives with cardiovascular disease, putting them at a higher risk of cardiovascular disease. Although randomized trials of ASA in ET patients are lacking in general, current treatment guidelines recommend that intermediate-risk ET patients should receive low-dose ASA.

High-risk ET patients, as defined by the DGHO and ELN, are those patients that are aged over 60 years and/or have experienced thrombosis/thromboembolism or severe hemorrhage previously, and/or in whom platelet counts have exceeded $1500 / \mathrm{nL}^{12}$ These patients are at a higher risk of developing thrombosis/thromboembolism and/or severe bleeding and therefore require cytoreductive therapy, in addition to ASA. Another reason to initiate cytoreductive therapy in such patients is the presence of significant bleeding risk due to platelet dysfunction and/or acquired von Willebrand syndrome (AVWS), ${ }^{31}$ as defined by a von Willebrand factor (VWF):ristocetin cofactor activity/VWF antigen ratio of $<0.7$ (see below and review in Appelmann et $\mathrm{al}^{31}$ ). Both platelet dysfunction and AVWS are more prevalent in patients with excessive than normal platelet counts but can occur even in patients with normal platelet counts. ${ }^{32}$ Therefore, I routinely perform screening for AVWS in MPN patients before initiating antiplatelet treatment. Nevertheless, it should be kept in mind that AVWS may be due to deficiency in high-molecular weight VWF multimers, and this requires confirmation by multimeric analysis of VWF. Finally, the presence of an AVWS does not predict bleeding, thus, the decision whether or not to withhold ASA is based primarily on clinical judgment of the bleeding risk, taking all of the above factors into account.

Hydroxyurea and anagrelide are approved first-line options for the treatment of high-risk ET. IFNa therapy is off-label, but is recommended in young patients, especially women who are or wish to become pregnant. Busulfan is a possible second- or third-line option, particularly in older 
patients. In addition, the following recommendations for antiplatelet and anticoagulant therapy exist.

ET patients with previous or concurrent arterial thrombosis/thromboembolism should receive standard management as those without ET. Life-long ASA as secondary prophylaxis is required. Exceptional cases may necessitate VKA treatment, such as those with recurrent arterial thrombosis/thromboembolism despite antiplatelet agents or those with APS. In patients with ET and myocardial infarction undergoing percutaneous coronary intervention with dualplatelet inhibition, using ASA and clopidogrel, there was no difference in the responsiveness to these two drugs when compared with non-ET patients. ${ }^{33}$ However, ASA-insensitive thromboxane biosynthesis has been described in ET patients, assessed by thromboxane B2 determination ex vivo, ${ }^{34}$ most likely due to accelerated production of new platelets. This was also confirmed in a second study. ${ }^{35}$ Both twice-daily instead of once-daily ASA and/or cytoreductive therapy led to inhibition of cyclooxygenase- 1 and platelet aggregation in vitro, ${ }^{34}$ but it is still unclear whether twice-daily ASA can safely decrease the risk of thrombosis in ET patients. Therefore, twice-daily ASA can be considered but is not currently recommended in patients with ET requiring ASA.

Importantly, patients with a high bleeding risk, including those with a VWF:ristocetin cofactor activity of less than $30 \%$ should not receive ASA, until their bleeding risk is lowered, e. g., by cytoreductive therapy. ${ }^{36}$ When used in combination with anagrelide, antiplatelet agents must be used with caution due to the higher bleeding risk. ${ }^{37}$

ET patients with previous or concurrent venous thrombosis/ VTE should be treated as their counterparts without ET. Again, anticoagulant treatment must be used with caution due to the higher bleeding risk, especially in patients with AVWS or those concomitantly treated with anagrelide, which has been shown to carry an elevated risk of bleeding when combined with antiplatelet agents. ${ }^{37}$ The duration of anticoagulant therapy in ET is still unsettled, particularly in light of the lower incidence of thrombosis/thromboembolism than in PV. However, in the absence of firm data, the same caution against co-administration of antiplatelet and anticoagulant treatment and the same duration of anticoagulant treatment are recommended as stated above for PV. ${ }^{24}$

Recently, a new scoring system, the so-called "IPSET-thrombosis" score, ${ }^{38}$ has retrospectively been defined to classify patients with ET according to their individual risk of thrombosis. According to this score, patients receive 1 point each in the case of an age over 60 years or cardiovascular risk factors, and 2 points each in the case of previous thrombosis or JAK2 V617F positivity. A sum of $0-1$ points, 2 , or $\geq 3$ points defines low, intermediate, or high thrombosis risk, respectively. ${ }^{38}$ The National Comprehensive Cancer Network (NCCN) guidelines recommend ASA to be "considered to reduce the risk of thrombotic complications for patients with very low risk, low-risk, or intermediate-risk ET" according to the IPSETthrombosis score. ${ }^{21}$ It is conceivable that, in the future, we will increasingly use biologic factors such as JAK2 V617F positivity for our therapeutic decisions, e.g., ASA use in JAK2 V617Fpositive ET, as has been proposed. ${ }^{39}$ However, most treatment recommendations have not yet firmly incorporated the IPSETthrombosis risk subgroups.

\section{Primary Myelofibrosis and Post-PV-MF and Post-ET-MF}

MPNs with myelofibrosis are further categorized into the following subtypes: prefibrotic PMF (pre-PMF), overt PMF, post-PV-MF, and post-ET-MF. For each of these subtypes, specific diagnostic criteria have been established. However, since PMF has only recently been subdivided into pre-PMF and overt PMF (WHO 2016 ${ }^{1}$ ), less information is available on these individual subtypes. However, it is clear for all four MF subtypes that patients with MF have a shorter survival as compared with age-matched controls and that they suffer from more severe symptoms, particularly weight loss, splenomegaly-related early satiety, and anemia-associated symptoms. ${ }^{2}$ It is less clear to what extent these patients are at risk for thrombosis/thromboembolism and bleeding. A recent analysis of the German MPN registry found considerable differences between PMF, post-PV-MF, and post-ET-MF for the rate of thrombosis/thromboembolism and severe bleeding. ${ }^{3}$

Risk scoring in MF, including the Dynamic International Prognostic Scoring System (DIPSS), is based upon clinical and molecular parameters and predicts survival. For PMF, several scores have been designed and validated, ${ }^{40,41}$ but the DIPSS score is used most commonly for therapy stratification. ${ }^{41}$ Based on this score, intermediate-2 (int-2) and high-risk MF patients should undergo eligibility screening for allogeneic SCT (alloSCT). If allo-SCT is not performed, symptomatic MF patients can be treated with ruxolitinib to alleviate their symptoms ${ }^{42}$ and possibly enhance survival. ${ }^{43}$ In patients with post-PV- or postET-MF, the Myelofibrosis Secondary to PV and ET-Prognostic Model (MYSEC score) should be calculated, ${ }^{44}$ and those patients with an int-2 or high-risk MYSEC score should be treated in the same way as described above for PMF patients.

No general recommendation for ASA use or anticoagulant treatment is available for MF patients. Thus, these patients are being treated on an empirical basis, with pre-PMF patients most often being treated according to the ET algorithm, due to the similarity of risk factors in pre-PMF and ET. Since thrombosis/thromboembolism and bleeding represent significant risks in MF (up to $62 \%$ of patients with post-PV-MF had experienced a thromboembolic episode ${ }^{3}$ ), careful screening for cardiovascular risk factors and thrombosis/thromboembolism is necessary in all MF patients. Additional caution for bleeding events is warranted in patients with MF-associated hematopoietic insufficiency, particularly in thrombocytopenic patients, and those with AVWS (see above for ET). ${ }^{31}$

\section{Concluding Remarks}

Patients with MPNs are at high risk of developing thromboembolic/thrombotic complications. At the same time, they are also prone to severe bleeding. These risks are influenced by age and the presence of additional cardiovascular risk factors, but also by the MPN subtype and the genetic mutational profile. Thus, first of all, a correct diagnosis must be established, and all 
relevant medical history, concomitant medication, and family history need to be taken into account. Then, disease-specific risk group allocation needs to be performed, including calculation of scores for overall survival (such as DIPSS and MYSEC scores in primary and secondary myelofibrosis) and for thrombosis-free survival (e.g., conventional risk factor assessment in PV or ET and IPSET-thrombosis score in ET). The risk group allocation for the individual patient helps to decide upon whether and which type of treatment is indicated. Both primary prophylaxis and secondary prophylaxis approaches for thrombosis/thromboembolism and severe hemorrhage are established for specific disease scenarios, and these should be initiated and combined with cytoreductive therapy as needed, according to current national and international treatment recommendations $^{12}$ (www.onkopedia.com/de) or guidelines. ${ }^{21}$ Finally, since MPNs are chronic diseases, regular monitoring of the thromboembolic/thrombotic risk as well as their risk of bleeding is necessary to maintain an optimal survival span and quality of life of our patients.

\section{Conflicts of Interest}

S.K. reports research funding from Novartis, Janssen, AOP Orphan Pharmaceuticals AG, and Bristol-Myers Squibb, as well as consultancy honoraria from Novartis, Incyte/ Ariad, Bristol-Myers Squibb, AOP Orphan Pharmaceuticals AG, Pfizer, Celgene, Bayer, Roche, CTI, and Shire.

\section{References}

1 Arber DA, Orazi A, Hasserjian R, et al. The 2016 revision to the World Health Organization classification of myeloid neoplasms and acute leukemia. Blood 2016;127(20):2391-2405

2 Emanuel RM, Dueck AC, Geyer HL, et al. Myeloproliferative neoplasm (MPN) symptom assessment form total symptom score: prospective international assessment of an abbreviated symptom burden scoring system among patients with MPNs. J Clin Oncol 2012;30(33):4098-4103

3 Kaifie A, Kirschner M, Wolf D, et al; Study Alliance Leukemia (SAL). Bleeding, thrombosis, and anticoagulation in myeloproliferative neoplasms (MPN): analysis from the German SAL-MPN-registry. J Hematol Oncol 2016;9:18

4 How J, Zhou A, Oh ST. Splanchnic vein thrombosis in myeloproliferative neoplasms: pathophysiology and molecular mechanisms of disease. Ther Adv Hematol 2017;8(03):107-118

5 Cerletti C, Tamburrelli C, Izzi B, Gianfagna F, de Gaetano G. Plateletleukocyte interactions in thrombosis. Thromb Res 2012;129(03): 263-266

6 Barbui T, Carobbio A, Finazzi G, et al; AGIMM and IIC Investigators. Inflammation and thrombosis in essential thrombocythemia and polycythemia vera: different role of C-reactive protein and pentraxin 3. Haematologica 2011;96(02):315-318

7 Edelmann B, Gupta N, Schnoeder TM, et al. JAK2-V617F promotes venous thrombosis through $\beta 1 / \beta 2$ integrin activation. J Clin Invest 2018;128(10):4359-4371

8 Zhang W, Qi J, Zhao S, et al. Clinical significance of circulating microparticles in $\mathrm{Ph}^{-}$myeloproliferative neoplasms. Oncol Lett 2017;14(02):2531-2536

9 Falanga A, Marchetti M, Evangelista V, et al. Polymorphonuclear leukocyte activation and hemostasis in patients with essential thrombocythemia and polycythemia vera. Blood 2000;96(13): 4261-4266

10 Kroll MH, Michaelis LC, Verstovsek S. Mechanisms of thrombogenesis in polycythemia vera. Blood Rev 2015;29(04):215-221
11 Wolach O, Sellar RS, Martinod K, et al. Increased neutrophil extracellular trap formation promotes thrombosis in myeloproliferative neoplasms. Sci Transl Med 2018;10(436):eaan8292

12 Barbui T, Barosi G, Birgegard G, et al; European LeukemiaNet. Philadelphia-negative classical myeloproliferative neoplasms: critical concepts and management recommendations from European LeukemiaNet. J Clin Oncol 2011;29(06):761-770

13 Girodon F, Bonicelli G, Schaeffer C, et al. Significant increase in the apparent incidence of essential thrombocythemia related to new WHO diagnostic criteria: a population-based study. Haematologica 2009;94(06):865-869

14 Stein BL, Oh ST, Berenzon D, et al. Polycythemia vera: an appraisal of the biology and management 10 years after the discovery of JAK2 V617F. J Clin Oncol 2015;33(33):3953-3960

15 Tefferi A, Rumi E, Finazzi G, et al. Survival and prognosis among 1545 patients with contemporary polycythemia vera: an international study. Leukemia 2013;27(09):1874-1881

16 Landolfi R, Marchioli R, Kutti J, et al; European Collaboration on Low-Dose Aspirin in Polycythemia Vera Investigators. Efficacy and safety of low-dose aspirin in polycythemia vera. $\mathrm{N}$ Engl J Med 2004;350(02):114-124

17 Marchioli R, Finazzi G, Specchia G, et al; CYTO-PV Collaborative Group. Cardiovascular events and intensity of treatment in polycythemia vera. N Engl J Med 2013;368(01):22-33

18 Barbui T, Vannucchi AM, Finazzi G, et al. A reappraisal of the benefit-risk profile of hydroxyurea in polycythemia vera: a propensity-matched study. Am J Hematol 2017;92(11):1131-1136

19 Gisslinger H, Zagrijtschuk O, Buxhofer-Ausch V, et al. Ropeginterferon alfa-2b, a novel IFN $\alpha-2 b$, induces high response rates with low toxicity in patients with polycythemia vera. Blood 2015; 126(15):1762-1769

20 Vannucchi AM, Kiladjian JJ, Griesshammer M, et al. Ruxolitinib versus standard therapy for the treatment of polycythemia vera. N Engl J Med 2015;372(05):426-435

21 Mesa RA, Jamieson C, Bhatia R, et al. NCCN Guidelines Insights: Myeloproliferative Neoplasms, Version 2.2018. J Natl Compr Canc Netw 2017;15(10):1193-1207

22 Vannucchi AM, Barbui T, Cervantes F, et al; ESMO Guidelines Committee. Philadelphia chromosome-negative chronic myeloproliferative neoplasms: ESMO Clinical Practice Guidelines for diagnosis, treatment and follow-up. Ann Oncol 2015;26(Suppl 5):v85-v99

23 De Stefano V, Za T, Rossi E, et al; GIMEMA CMD-Working Party. Recurrent thrombosis in patients with polycythemia vera and essential thrombocythemia: incidence, risk factors, and effect of treatments. Haematologica 2008;93(03):372-380

24 Kreher S, Ochsenreither S, Trappe RU, et al; Haemostasis Working Party of the German Society of Hematology and Oncology; Austrian Society of Hematology and Oncology; Society of Thrombosis and Haemostasis Research. Prophylaxis and management of venous thromboembolism in patients with myeloproliferative neoplasms: consensus statement of the Haemostasis Working Party of the German Society of Hematology and Oncology (DGHO), the Austrian Society of Hematology and Oncology (ÖGHO) and Society of Thrombosis and Haemostasis Research (GTH e.V.). Ann Hematol 2014;93(12):1953-1963

25 De Stefano V, Finazzi G, Barbui T. Antithrombotic therapy for venous thromboembolism in myeloproliferative neoplasms. Blood Cancer J 2018;8(07):65

26 Klampfl T, Gisslinger H, Harutyunyan AS, et al. Somatic mutations of calreticulin in myeloproliferative neoplasms. N Engl J Med 2013;369(25):2379-2390

27 Barbui T, Thiele J, Gisslinger H, Finazzi G, Vannucchi AM, Tefferi A. The 2016 revision of WHO classification of myeloproliferative neoplasms: clinical and molecular advances. Blood Rev 2016;30(06):453-459

28 Barbui T, Thiele J, Passamonti F, et al. Survival and disease progression in essential thrombocythemia are significantly influenced by accurate morphologic diagnosis: an international study. J Clin Oncol 2011;29(23):3179-3184 
29 Alberio L. Do we need antiplatelet therapy in thrombocytosis? Pro. Diagnostic and pathophysiologic considerations for a treatment choice. Hamostaseologie 2016;36(04):227-240

30 Scharf RE. Do we need antiplatelet therapy in thrombocytosis? Contra. Proposal for an individualized risk-adapted treatment. Hamostaseologie 2016;36(04):241-260

31 Appelmann I, Kreher S, Parmentier S, et al. Diagnosis, prevention, and management of bleeding episodes in Philadelphianegative myeloproliferative neoplasms: recommendations by the Hemostasis Working Party of the German Society of Hematology and Medical Oncology (DGHO) and the Society of Thrombosis and Hemostasis Research (GTH). Ann Hematol 2016;95 (05):707-718

32 Tefferi A, Smock KJ, Divgi AB. Polycythemia vera-associated acquired von Willebrand syndrome despite near-normal platelet count. Am J Hematol 2010;85(07):545

33 Campo G, Valgimigli M, Carletti R, Fileti L, Ferrari R. Long-term outcome after percutaneous coronary intervention in patients with essential thrombocythemia. J Thromb Haemost 2009; 7(07): 1235-1238

34 Pascale S, Petrucci G, Dragani A, et al. Aspirin-insensitive thromboxane biosynthesis in essential thrombocythemia is explained by accelerated renewal of the drug target. Blood 2012;119(15): 3595-3603

35 Perrier-Cornet A, Ianotto JC, Mingant F, Perrot M, Lippert E, Galinat $H$. Decreased turnover aspirin resistance by bidaily aspirin intake and efficient cytoreduction in myeloproliferative neoplasms. Platelets 2018;29(07):723-728

36 Tefferi A, Barbui T. Polycythemia vera and essential thrombocythemia: 2015 update on diagnosis, risk-stratification and management. Am J Hematol 2015;90(02):162-173
37 Birgegård G, Besses C, Griesshammer M, et al. Treatment of essential thrombocythemia in Europe: a prospective long-term observational study of 3649 high-risk patients in the Evaluation of Anagrelide Efficacy and Long-term Safety study. Haematologica 2018;103(01):51-60

38 Barbui T, Finazzi G, Carobbio A, et al. Development and validation of an International Prognostic Score of thrombosis in World Health Organization-essential thrombocythemia (IPSET-thrombosis). Blood 2012;120(26):5128-5133, quiz 5252

39 Rumi E, Cazzola M. Diagnosis, risk stratification, and response evaluation in classical myeloproliferative neoplasms. Blood 2017; 129(06):680-692

40 Gangat N, Caramazza D, Vaidya R, et al. DIPSS plus: a refined dynamic international prognostic scoring system for primary myelofibrosis that incorporates prognostic information from karyotype, platelet count, and transfusion status. J Clin Oncol 2011;29(04):392-397

41 Passamonti F, Cervantes F, Vannucchi AM, et al. A dynamic prognostic model to predict survival in primary myelofibrosis: a study by the IWG-MRT (International Working Group for Myeloproliferative Neoplasms Research and Treatment). Blood 2010;115(09):1703-1708

42 Harrison C, Kiladjian JJ, Al-Ali HK, et al. JAK inhibition with ruxolitinib versus best available therapy for myelofibrosis. $\mathrm{N}$ Engl J Med 2012;366(09):787-798

43 Verstovsek S, Gotlib J, Mesa RA, et al. Long-term survival in patients treated with ruxolitinib for myelofibrosis: COMFORT-I and -II pooled analyses. J Hematol Oncol 2017;10(01):156

44 Passamonti F, Giorgino T, Mora B, et al. A clinical-molecular prognostic model to predict survival in patients with post polycythemia vera and post essential thrombocythemia myelofibrosis. Leukemia 2017;31(12):2726-2731 